\title{
A LIST OF THE FERNS AND FERN ALLIES OF NORTH AMERICA NORTH OF MEXICO, WITH PRINCIPAL SYNO- NYMS AND DISTRIBUTION.
}

\author{
By William R. Maxon, \\ Aid in Cryptogamic Botany, Division of Plants.
}

In all the literature relating to American ferns and the so-called "fern allies," there have hitherto appeared but two systematic treatises of sufficiently wide scope to include the entire territory of North America north of Mexico, namely, the Ferns of North America, by Prof. D. C. Eaton (1877-1880), and Our Native Ferns and Their Allies (in several editions, 1881-1900), by Prof. L. M. Underwood. The first of these, a monograph of two large quarto volumes, contains descriptions and colored figures of all species comprised in the groups known at that time as the orders Filices and Ophioglossaceae. The treatmen $\mathrm{n}$ is full but concise, the style clear, the figures for the most part excellent; and the work as a whole must be regarded as the foundation for all subsequent studies of North American ferns. Without its good influence it is unlikely that the present degree of progress could have been attained; but we should remember first of all that it represents a critical estimate of the groups as they were understood over twenty years ago, and that since its appearance there has elapsed a period marked by unexampled botanical activity and progress. There has been collected in the meantime abundant material of many species either entirely new or then represented in herbaria by mere fragments, and with the aid of these specimens careful studies have been accomplished in the light of which not only new specific names have been proposed, but many changes in the older definitions of species have been shown to be desirable. If, then, we recognize that there have been and must continue to be many departures from the treatment contained in Professor Eaton's great work, we may escape that extreme conservatism which occasionally manifests itself in opposing innovation of almost any sort, and which regards the setting aside of an opinion there expressed as a proceeding hardly within the bounds of propriety. 
The cost of Professor Eaton's volumes has been such, unfortunately, as to place them beyond the reach of a majority of fern students; and largely on this account it has remained for Professor Underwood's attractive little book to really popularize the study of ferns within the United States. The first edition of the latter appeared in 1881, and was entitled Our Native Ferns and How to Study Them. It contained 116 pages, the first half being devoted to chapters on the haunts, habits, and distribution of ferns, their morphology and structure, methods of study, and the like; the remaining portion comprising a systematic arrangement of the groups treated by Eaton in his larger work. The second edition, made necessary by a remarkable demand for the first, appeared the following year under the slightly emended title, Our Native Ferns and Their Allies, preserving the general scheme of the former volume, but extending the systematic treatment to include the Equisetaceae, Marsileaceae, Salviniaceae, Lycopodiaceae, Selaginellaceae, and Isoëtaceae, which had merely been listed in the first edition. The third edition appeared in 1888, being practically an enlargement of the preceding. The fourth was issued in 1893, and contained a number of nomenclatorial changes - notably the substitution of Dryopteris for Aspidium - to bring the nomenclature to the standard set by the "Rochester code." The fifth edition (1896), except for the addition of a few species, is practically like the fourth. The sixth and last, which appeared in June, 1900, is extensively remodeled in conformity with the author's views as set forth in part in a Review of the Genera of Ferns proposed prior to $1832 .^{1}$ The most notable changes have to do with matters of nomenclature, though the systematic arrangement is also considerably modified and the number of species increased. It is hardly to be supposed that further studies will not result in addi. tional changes; nevertheless nothing is more certain than that the present edition represents the most logical sequence of genera and the most reasonable estimate of our species that has yet been presented. Its general scheme has been followed closely in the preparation of the present paper.

Owing to the fact that Professor Underwood's is essentially a popular treatise, all citations are naturally and properly omitted. In the Ferns of North America, on the other hand, we have extensire but often incomplete bibliography under each species; but notwithstanding its incompleteness there has hitherto appeared no index of synonyms or compendium of any sort to take its place. The present list has been prepared, therefore, with the object of affording full citations for all the included species and for the more important synonyms. An especial effort has been made to insure the accurate citation of references to all original descriptions. It has been impossible to verify without exception every citation; but the number unverified is very

${ }^{1}$ Mem. Torr. Club $6: 247-283 . \quad 1899$. 
small. The changes in the nomenclature of certain groups have been so extensive during the past few years as to necessitate devoting considerable space to their synonymy; but a constant aim has been to avoid the burden of useless bibliography. Such vernacular names as are in general use have been admitted, following the correct scientific name of the species and in the order of their importance.

There is at least one other feature which has seemed to warrant the publication of this list in its present form, namely, the considerable amount of attention given nowadays to geographical distribution. Within the past twenty years we have had but three comprehensive lists relating to North American pteridophyta and dealing with this phase of fern study. The first of these, a Systematic Fern-list, was issued by Professor Eaton in 1880. Its scope is well explained in the supplementary title, A Classified List of the Known Ferns of the United States of America, with the Geographical Range of the Species. The disposition is practically that of the Ferns of North America. The second list, published by Mr. George E. Davenport in the Proceedings of the American Philosophical Society in 1883 , applies only to the United States and Alaska. It contains an enumeration of the ferns only, some comparative tables showing their distribution state by state, and an interesting discussion of the range of certain species. The third, issued in 1895, by the Linnæan Fern Chapter, as Linnæan Fern Bulletin No. 9, is a list of the species of Pteridophyta of North America north of Mexico, without synonymy, but with distribution briefly indicated. These and the numerous other lists and papers mentioned below have been consulted in the preparation of this paper; but the ranges herein given have been determined chiefly by careful examination of the material in the U.S. National Herbarium, the herbarium of Columbia University, the herbarium of the New York Botanical Garden, and, in certain genera, the D. C. Eaton herbarium at Yale University and the herbarium of the California Academy of Sciences. Few records unsubstantiated by specimens have been allowed. Many wrong identifications have been corrected, and the utmost care has been taken to eliminate doubtful records, whether of old or recent standing.

The following summary will be found to contain a large proportion of the more important lists or extended papers on the distribution and systematic relationship of our species:

1829. Synoptical Tables of the Ferns and Mosses of the United States. Lewis C. Beck in the American Journal of Science 15: 287-297.

1840. Acotyledonae [of North America]. Sir W. J. Hooker, Flora Boreali-Americana 2: $258-270$.

1843. Flowerless or Cryptogamous Plants [of New York State]. John Torrey, A Flora of the State of New York 2: 480-514.

1844. A Monography of the North American Species of Equisetum. Alexander Braun in the American Journal of Science 46: 81-91. 
1847. On the North American Species of Isoëtes and Marsilea. Alexander Braun in the American Journal of Science II. 3: 52-56.

1848. Notes on some Ferns of the United States. G. Kunze in the American Journal of Science II. 6: 80-89.

1859. [Equisetaceae, Filices, Lycopodiaceae, Hydropterides]. D. C. Eaton in Report on the United States and Mexican Boundary Survey $2^{1}:$ 233-236.

1860. Filices [of the Southern United States]. D. C. Eaton in Chapman, Flora of the Southern United States ed. 1. 585-599.

1864. Synopsis of Canadian Ferns and Filicoid Plants. George Lawson in Canadian Naturalist II. 1: 262-300.

1864. Notes on the Habitats and Varieties of some Canadian Ferns. David R. MeCord in Canadian Naturalist II. 1: 354-362.

1867. Filices [of the Northern United States]. D. C. Eaton in A. Gray, Manual of Botany of the Northern United States ed. 5. 655-672.

1870. [Review of Katharine M. Lyell's The Geographical Handbook of Ferns.] D. A. Watt in Canadian Naturalist II. $5: 343-349$.

1873. Checklist of the Ferns of North America north of Mexico. John Robinson.

1875. Geographical Distribution of the Ferns of North America. John H. Redfield in Bulletin of the Torrey Botanical Club 6: 1-7.

1876. Catalogue of the Davenport Herbarium. George E. Davenport.

1876. Catalogue of North American Ferns. William Edwards. [Ed. 4. The third
[E. The edition (1874) bears a slightly different title. A fifth edition appeared in 1879.]

1878. Ferns of Kentucky. John Williamson. [A local fern flora of 155 pages, containing illustrations of the species, and brief chapters on the structure, cultivation, collection, and study of ferns.]

1879. Catalogue of the "Davenport Herbarium" of North American Ferns . . . . George E. Davenport.

1879. Ferns of the Southwest. D. C. Eaton in Report U. S. Geographical Surveys West of One Hundredth Meridian 6: 301-340. [Volume 6 of this report is usually known as "Bot. Wheeler Survey." It bears date of 1878 , but appeared first in May, 1879.]

1880. Systematic Fern-list; a Classified List of the Known Ferns of the United States of America, with the geographical range of the Species. Daniel C. Eaton.

1880. Vascular Acrogens [of California]. D. C. Eaton in Watson and Brewer, Botany of California 2: 329-352.

1882. Ferns of the Pacific Coast, including Arizona. J. G. Lemmon.

1882. The Genus Isoëtes in North America. George Englemann in Transactions of the St. Louis Academy of Science 4: 358-390.

1882. Ferns of the West. Marcus E. Jones.

1883. Some Comparative Tables showing the distribution of Ferns in the United States of America. George E. Davenport in Proceedings of the American Philosophical Society 20: 605-612.

1883. Catalogue of the Davenport Herbarium. Supplement. George E. Davenport. 1885. Canadian Filicineae. John Macoun and T. J. W. Burgess in Proceedings and Transactions of the Royal Society of Canada $2^{4}: 163-226$.

1887. Notes on the American Species of Marsilea. L. M. Underwood and O. F. Cook in Bulletin of the Torrey Botanical Club 14: 89-94.

1887. Recent Additions to the Canadian Filicineae. . . . . T. J. W. Burgess in Proceedings and Transactions of the Royal Society of Canada 44: 9-18.

1888. The distribution of Isoëtes. L. M. Underwood in Botanical Gazette 13: 89-94.

1889. The Fern Flora of Canada. George Lawson. [This excellent work was reissued later in the same year under the title A School Fern Flora of Canada as an Appendix (pp. 221-251) to How Plants Grow, by Asa Gray]. 
1890. Catalogue of Canadian Plants. Part V. Acrogens. John Macoun.

1890. Vascular Acrogens or Pteridophytes [of the Northern United States]. D. C.

Eaton in A. Gray, Manual of Botany of the Northern United States ed. 6. $675-701$.

1895. Ferns and Evergreens of New England. Edward Knobel.

1895. The Pteridophyta of North America, north of Mexico. Linnæan Fern Bulletin No. 9, Willard N. Clute, editor.

1896. The Ferns and Fern Allies of New England. Raynal Dodge. [An excellent descriptive work of viii +52 pages. $]$

1896. Pteridophyta [of the Northern United States, Canada . . . . ]. L. M. Underwood in Britton and Brown, Illustrated Flora of the Northern United States, Canada ... . 1: 1-48.

1896. Ferns of Iowa and their Allies. T. J. Fitzpatrick.

1897. A Revision of the North American Species of Ophioglossum. Elizabeth G. Britton in Bulletin of the Torrey Botanical Club 24: 545-559.

1898. Selaginella rupestris and its Allies. L. M. Underwood in Bulletin of the Torrey Botanical Club 25: 125-133.

1898. American Ferns: I; the ternate species of Botrychium. L. M. Underwood in Bulletin of the Torrey Botanical Club 25: 521-541.

1899. How to Know the Ferns. Frances Theodora Parsons. [An extremely popular account of the ferns of the northeastern United States; pp. 215.]

1900. A Review of the Species of Lycopodium of North America. F. E. Lloyd and L. M. Underwood in Bulletin of the Torrey Botanical Club 27: 147-168.

1900. The Genus Isoëtes in New England. A. A. Eaton in Fernwort Papers 1-16.

The files of the Torrey Botanical Club, especially the early volumes, present an unusually large proportion of interesting short papers and notes. Special mention should be given of a long series by Mr. George E. Davenport, beginning in the sixth volume (1875), and embracing descriptions of many new species and notes on the discovery and distribution of many others. In the same journal Professor Eaton began with the fourth volume (1873) a series entitled New and Little-known Ferns of the United States, which extended to the tenth volume (1883). The files of the Botanical Gazette, the American Naturalist, the American Journal of Science, and several other serials are replete with references to our American species, as are also in many cases the publications of the various state geological surveys. The only journal devoted exclusively to the study of pteridophyta is The Fern Bulletin, published in Binghamton, New York, and beginning with 1901 its ninth volume. It was established in 1893 as the Linnæan Fern Bulletin, and from that time on has constituted the official organ of the Linnæan Fern Chapter, an organization of fern students becoming world-wide. Descriptions of many new species have appeared in this journal during the past few years.

The greater portion of the verification of citations following has been accomplished in the Library of Congress, the libraries of the Smithsonian Institution and the U. S. National Museum, and in the library of the Department of Agriculture. To Prof. E. L. Greene the author is indebted for many courtesies, and particularly for the use of his extensive library; to $\mathrm{Mr}$. Willard $\mathrm{N}$. Clute for the suggestion 
of the undertaking itself and for considerable assistance in its early stages; to Prof. L. M. Underwood and Mr. George E. Davenport for the completion or verification of many references, and especially to the former for many critical observations; to Mr. B. D. Gilbert for courtesies extended, especially in the study of specimens in his excellent private herbarium; to Mr. Frederick V. Coville, Mr. O. F. Cook, Dr. J. N. Rose, and Mr. Charles Louis Pollard for numerous helpful suggestions and criticisms; to Mr. Alvah A. Eaton for revising the Isoëtaceae and Equisetaceae; and to several others who have courteously assisted by contributing specimens and data or by supplying references otherwise unavailable.

\section{PTERIDOPHYTA Cohn.}

Family I. OPHIOGLOSSACEAE Presl, Tent. Pterid. 10. 1836.

\section{OPHIOGLOSSUM L. Sp. Pl. 1062. 1753.}

Ophioglossum vulgatum L. Sp. Pl. 1062. 1753. Adder's-Tongue.

Quebec and Ontario, south to Florida. Also in California.

Ophioglossum engelmanni Prantl, Jahrb. Kön. Bot. Gart. Berlin 3 : 318 . pl. \%.f. $1 \% .1884$.

Virginia, Kentucky, Indiana, and Missouri, southwest to Louisiana, Texas, and Arizona.

Ophioglossum arenarium E. G. Britton, Bull. Torr. Club 24:555. pl. 318. pl. 319. f. 3. 1897.

Holly Beach, New Jersey. Rockingham County, New Hampshire (A. A. Eaton).

Ophioglossum californicum Prantl, Jahrb. Kön. Bot. Gart. Berlin 3:315. pl. \%.f. 11. 1884 .

Near San Diego, California (Cleveland \& Parry; Pringle).

0phioglossum alaskanum E. G. Britton, Bull. Torr. Club 24 : 556. pl. 319. f. 5. 1897.

Unalaska Island, Alaska (Turner).

0phioglossum pusillum Nutt. Gen. 2 : 248. 1818.

Ophioglossum nudicaule Sturm; Mart. Fl. Bras. $1^{2}: 144$. 1840, not L. f.

South Carolina to Florida, west to Louisiana. Also in Arizona.

Ophioglossum crotalophoroides Walt. Fl. Car. 256. 1788.

Ophioglossum bulbosum Michx. Fl. Bor. Am. 2 : 276. 1803.

South Carolina and Florida; Alabama, westward to Texas.

CHEIR0GL0SSA Presl, Abh. Kön. Böhm. Gesell. Wiss. V. $4: 316.1847$.

Cheiroglossa palmata (L.) Presl, Abh. Kön. Böhm. Gesell. Wiss. V. 4:317. 1847.

Ophioglossum palmatum L. Sp. Pl. 1063. 1753.

Florida. 
BOTRYCHIUM Sw. Schrad. Journ. Bot. 1800² : 8. 1801.

Botrychium pumicola Coville in Underw. Our Native Ferns ed. 6. 69. 1900. Crater Lake, Oregon (Coville \& Applegate).

Botrychium tenebrosum A. A. Eaton, Fern Bull. 7: 8. 1899.

New Hampshire to Connecticut, Long Island (New York) and central New York.

Botrychium simplex E. Hitchcock, Am. Journ. Sci. 6 : 103. p7. 8. 1823. SNAKE-TONGUE.

New England to Quebec, west to Wyoming, California, and Oregon.

Botrychium lunaria (L.) Sw. Schrad. Journ. Bot. 1800²:110. 1801. MoONwort.

Osmunda lunaria L. Sp. Pl. 1064. 1753.

Newfoundland to Connecticut, central New York, Michigan, and Minnesota. Also in Alaska, and south in the mountains to Montana, Colorado, Utah, and California.

Botrychium boreale (Fries) Milde, Bot. Zeit. 15: 880. 1857.

Botrychium lunaria var. boreale. Fries, Herb. Normale 16 : 85 .

Unalaska, Alaska.

Botrychium neglectum Wood, Class-book ed. 2. 816. 1860.

Botrychium matricariaefolium of American authors, not A. Br.; Döll. Rhein. Fl. 24. 1843.

Nova Scotia and New Brunswick to Maryland, Ohio, and South Dakota. Alaska to Washington.

Botrychium matricariae (Schrank) Spreng. Syst. Veg. 4 : 23. 1827.

Osmunda matricariae Schrank, Baier. Fl. 2 : 419. 1789.

Botrychium rutaceum Sw. Schrad. Journ. Bot. 1800² : 110. 1801.

Botrychium matricarioides Willd. Sp. Pl. 5 : 62.1810.

Botrychium rutaefolium A. Br. in Döll. Rhein. Fl. 24. 1843.

Labrador to northern New England and central New York.

Botrychium biternatum (Lam.) Underw. Bot. Gaz. 22 : 407. pl. 21. 1896. Osmunda biternata Lam. Encyc. $4: 650.1797$.

Botrypus lunarioides Michx. Fl. Bor. Am. 2 : 274. 1803.

Botrychium lunarioides Sw. Syn. Fil. 172. 1806, not Gray.

Botrychium ternatum var. lunarioides D. C. Eaton, Ferns N. Am. 1 : 148. pl. 20. f. 3. 1878.

South Carolina to Florida and Louisiana.

Botrychium dissectum Spreng. Anleit. Kennt. Gewächse ed. 1. $3: 172$. 1804. Grape fern. Rattlesnake fern.

Botrychium ternatum var. dissectum D. C. Eaton, Ferns N. Am. $1: 150 . p l .20$. f. 1. 1878 .

Maine to Virginia, Kentucky, Indiana, and Ohio.

Botrychium obliquum Muhl.; Willd. Sp. Pl. 5:63. 1810. Grape Fern. RatTlesNake FERN.

Botrychium ternatum var. obliquum D. C. Eaton, Ferns N. Am. $1: 149$. pl. 20. f. 2. 1878.

New Brunswick to Florida, Indiana, and Minnesota.

Proc. N. M. vol. xxiii- 40 
Botrychium obliquum intermedium (D. C. Eaton) Underw. Our Native

Ferns ed. 6. 72. 1900. Grape FERn.

Botrychium ternatum var. australe sukvar. intermedium D. C. Eaton, Ferns N. Am. $1: 149 . p l .20 a$ in part 187

New England and New York.

Botrychium silaifolium Presl, Rel. Haenk. 1 : 76. 1830.

Botrychium ternatum var. australe D. C. Eaton, Ferns N. Am. 1:149. pl. $20 a$ in part. 1878. Not Botrychium australe R. Br.

California, Oregon, and British Columbia.

Botrychium coulteri Underw. Bull. Torr. Club 25 : 537. 1898.

Wyoming, the Yellowstone National Park, Montana, and Idaho.

Botrychium occidentale Underw. Bull. Torr. Club 25 : 538. 1898. WestERN MOONWORT.

Oregon, Washington, and British Columbia.

Botrychium lanceolatum (S. G. Gmel.) Ångs. Bot. Notiser 1854 : 68. 1854.

Osmunda lanceolata S. G. Gmel. Nov. Comm. Acad. Sci. Petrop. 12: 516. 1768.

Nova Scotia to New Jersey, Pennsylvania, Ohio, Michigan, and Alaska. Also in British Columbia, Washington, and Colorado.

Botrychium virginianum (L.) Sw. Schrad. Journ. Bot. 1800² : 111. 1801. Rattlesnake Fern. Grape Fern.

Osmunda virginiana L. Sp. Pl. 1064. 1753.

Botrychium gracile Pursh, Fl. Am. Sept. 2 : 656. 1814.

Nova Scotia and Labrador to British Columbia and Washington, south to Arizona, Texas, and Florida.

Family II. HYMENOPHYLLACEAE Gaud. in Bot. Freyc. Voy. 262. 1826.

TRICHOMANES L. Sp. Pl. 1097. 1753.

Trichomanes petersii A. Gray, Am. Journ. Sci. II. 15 : 326. 1853. Peters' filmy Fern.

Alabama: Winston County (Peters, Underwood); Black Creek Falls, Etowah County (Mohr; Pollard \& Maxon); Marion County (E. A. Smith).

Trichomanes radicans Sw. Fl. Ind. Occ. $3: 1736.1806 . \quad$ Bristle-Fern. Kentucky to Alabama and Florida.

Family. III. SCHIZAEACEAE Reichenb. Consp. 39. 1828.

SChizaea J. E. Smith, Mém. Acad. Roy. Sci. Turin $5: 419$. 1793.

Schizaea pusilla Pursh, Fl. Am. Sept. 2 : 657. 1814. Curly-grass.

New Jersey, Nova Scotia, and Newfoundland. Rare and local. 
LYGoDIUM Sw. Schrad. Journ. Bot. $1800^{2}: 106.1801$.

Lygodium palmatum (Bernh.) Sw. Syn. Fil. 154. 1806. Chimbing Fern. Hartford fern. Creeping fern. Windsor fern. Alice's FERN.

Gisopteris palmata Bernh. Schrad. Journ. Bot. $1800^{2}$ : 129. 1801.

New Hampshire and Massachusetts to Florida. Also in Kentucky and Tennessee. Mainly coastal.

ORNITHoPtERIS Bernh. Schrad. Neues Journ. Bot. 1²: 40. 1806.

Ornithopteris adiantifolia (L.) Bernh. Schrad. Neues Journ. Bot. $1^{2}$ : 50. pl. 3. f. 15. b. 1806.

Osmunda adiantifolia L. Sp. Pl. 1065. 1753.

Anemia adiantifolia Sw. Syn. Fil. 157. 1806.

Florida.

Ornithopteris mexicana (Klotzsch) Underw. Our Native Ferns ed. 6. 76. 1900.

Aneimia mexicana Klotzsch, Linnaea 18 : 526. 1844.

Western Texas.

Family IV. OSMUNDACEAE R. Br. Prodr. Fl. Nov. Holl. 1: 161. 1810.

OSMUNDA L. Sp. Pl. 1063. 1753.

0smunda regalis L. Sp. Pl. 1065. 1753. Royal Fern. Flowering Fern. QUEen FERN. WATER FERN. BUCK-HORN FERN.

Newfoundland to Florida, west to Mississippi, Nebraska, and Saskatchewan.

0smunda claytoniana L. Sp. Pl.1066. 1753. Interrupted Fern. ClayTON'S FERN.

Osmunda interrupta Michx. Fl. Bor. Am. 2 : 273. 1803.

Newfoundland to Minnesota, south to North Carolina, Kentucky, and Missouri.

0smunda cinnamomea L. Sp. Pl. 1066. 1753. Cinnamon fern. Brakes.

WoOLly Flowering-FERn. Fiddle-HeAds.

Labrador to Newfoundland, Nova Scotia, and Minnesota, south to Florida, Louisiana, and New Mexico. The form known as var. frondosa is found with the type.

Family V. CERATOPTERIDACEAE Underw. Our Native Ferns ed. 6. 78. 1900 .

CERATOPTERIS Brong. Bull. Soc. Philom. 1821: 184. 1821.

Ceratopteris thalictroides (L.) Brong. Bull. Soc. Philom. 1821: 186. pl. [1]. 1821. Floating FERN.

Acrostichum thalictroides L. Sp. Pl. 1070. 1753.

Florida. 
Family VI. POLYPODIACEAE Presl, Tent. Pterid. 167. 1836.

ACROSTICHUM L. Sp. Pl. 1067. 1753.

Acrostichum aureum L. Sp. Pl. 1069. 1753.

Southern Florida.

Acrostichum lomarioides Jenm. Bull. Bot. Dept. Jam. II. 5 : 154.1898.

Chrysodium lomarioides Jenm. Timehri 4 : 314. 1885.

Southern Florida.

POLYPODIUIM L. Sp. Pl. 1082. 1753.

Polypodium vulgare L. Sp. Pl. 1085. 1753. Polypody. Polypod.

Labrador and Newfoundland to Georgia, Alabama, Missouri, Manitoba, and Keewatin. Probably has a wider range toward the northwest.

Polypodium vulgare deceptum Maxon, nom. nov.

Polypodium vulgare forma biserrata Millsp. W. Va. Exp. Sta. Bull. 24; 479. 1892.

Not Polypodium biserratum Mart. \& Gal.

Polypodium vulgare oreophilum Maxon in Morris, Proc. Biol. Soc. Wash. 13 : 174.

1900. Not Polypodium orophilum Gandoger.

West Virginia.

Polypodium vulgare occidentale Hook. Fl. Bor. Am. 2 : 258. 1840. California to Alaska, along the coast.

Polypodium hesperium Maxon, Proc. Biol. Soc. Wash. 13 : 200. 1900. Arizona to Washington, British Columbia, and Montana.

Polypodium falcatum Kellogg, Proc. Cal. Acad. Sci. 1: 20. 1854.

Polypodium glycyrrhiza D. C. Eaton, Am. Journ. Sci. II. 22 : 138. 1856.

California to British Columbia and Alaska.

Polypodium plumula H. \& B.; Willd. Sp. Pl. 5 : 178. 1810.

Florida.

Polypodium pectinatum L. Sp. Pl. 1085. 1753.

Southern Florida.

Polypodium polypodioides (L.) A. S. Hitchcock, Rep. Mo. Bot. Gard. $4: 156$. 1893. Gray polypody. Resurrection fern. Tree fern. Acrostichum polypodioides L. Sp. Pl. 1068. 1753.

Polypodium incanum Sw. Fl. Ind. Occ. 3 : 1645. 1806.

Virginia to Florida, west to Iowa, Kansas, and Texas. On driftwood, Staten Island, New York (Bastedo).

Polypodium thysanolepis A. Br. in Klotzsch, Linnaea 20 : 392.1847.

Huachuca Mountains, Arizona (Lemmon).

Polypodium californicum Kaulf. Enum. Fil. 102. 1824.

Polypodium intermedium Hook. \& Arn. Bot. Beech. Voy. 405. 1841, not Muhl. California. Extremely variable, presenting a number of forms.

Polypodium scouleri Hook. \& Grev. Icon. Fil. 1: pl. 56. 1829.

Polypodium carnosum Kellogg, Proc. Cal. Acad. Sci. 2 : 88. f. 24. 1861.

Polypodium pachyphyllum D. C. Eaton, Am. Journ. Sci. II. 22 : 138. 1856.

California to British Columbia. 
PHLEBODIUM J. Sm. Journ. Bot. 4 : 58. 1842.

Phlebodium aureum (L.) J. Sm. Journ. Bot. 4: 59.1842. Golden POLYPODY. RABBIT'S-FOOT FERN.

Polypodium aureum L. Sp. Pl. 1087. 1753.

Florida.

CAMPLYONEURON Presl, Tent. Pterid. 189. 1836.

Camplyoneuron phyllitidis (L.) Presl, Tent. Pterid. 190. pl. \%. f. 18. 1836.

Polypodium phyllitidis L. Sp. Pl. 1083. 1753.

Florida.

PHYMATODES Presl, Tent. Pterid. 195. 1836.

Phymatodes swartzii (Baker) Underw. Our Native Ferns ed. 6. 84. 1900. Polypodium serpens Sw. Fl. Ind. Occ. 3 : 1633. 1806, not Forster.

Polypodium swartzii Baker in Hook. \& Baker, Syn. Fil. ed. 1. 357. 1868.

Key Largo, Florida (Curtiss; Pollard, Morris, \& Collins).

GYMNOPTERIS Bernh. Schrad. Journ. Bot. 1799¹ : 297.1799.

Gymnopteris hispida (Mett.) Underw. Our Native Ferns ed. 6. 84. 1900.

Gymnogramme hispida Mett. in Kuhn, Linnaea $36: 72$. 1869-70.

Texas to Arizona.

Gymnopteris triangularis (Kaulf.) Underw. Our Native Ferns ed. 6. 84. 1900 .

Gymnogramma triangulare Kaulf. Fnum. Fil. 73. 1824.

California to British Columbia. Also in Arizona.

Notholaena R. Br. Prodr. Fl. Nov. Holl. $1: 145.1810$.

Notholaena sinuata (Sw.) Kaulf. Enum. Fil. 135. 1824. Acrostichum sinuatum Sw. Syn. Fil. 14. 1806.

Texas to Arizona.

Notholaena ferruginea Desv. Journ. Bot. Appl. 1 : 92.1813.

Cincinalis ferruginea Desv. Mag. Gesell. Nat. Fr. Berlin 5 : 311. 1811.

Texas to Arizona.

Notholaena parryi D. C. Eaton, Am. Nat. 9 : 351. 1875.

Arizona and southern Utah to California.

Notholaena newberryi D. C. Eaton, Bull. Torr. Club 4:12.1873. CotTON FERN.

Southern California.

Notholaena aschenborniana Klotzsch, Linnaea $20: 417.1847$.

Huachuca Mountains (Lemmon) and Santa Rita Mountains (Pringle), Arizona; Texas (Drummond).

Notholaena candida (Mart. \& Gal.) Hook. Sp. Fil. 5 : 110. 1864. Cheilanthes candida Mart. \& Gal. Mém. Acad. Brux. 155: 73. pl. 20. 1842, in part. Southern Texas and New Mexico 
Notholaena cretacea Liebm. Mex. Breg. 64. 1849.

Southern California and Arizona.

Notholaena hookeri D. C. Eaton in U. S. Geog. Surv. W. 100th Merid. 6 : 308. pl. 30. 1879.

Texas to Arizona.

Notholaena grayi Davenp. Bull. Torr. Club 7 : 50. 1880.

Southeastern Arizona to Texas.

Notholaena lemmoni D. C. Eaton, Bull. Torr. Club 7 : 63. 1880. Arizona.

Notholaena schaffneri (Fourn.) Underw.; Davenp. Garden \& Forest $4: 519.1891$.

Aleuritopteris schaffneri Fourn. Bull. Bot. Soc. France $27: 328.1880$,

Notholaena nealleyi Seaton, Contrib. U. S. Nat. Herb. 1 : 61.1890.

Western Texas.

Notholaena nivea Desv. Journ. Bot. Appl. 1 : 93.1813.

Arizona and New Mexico.

Notholaena dealbata (Pursh) Kunze, Am. Journ. Sci. II. 6: 82.1848.

Cheilanthes dealbata Pursh Fl. Am. Sept. 2 : 671. 1814.

Notholaena nivea var. dealbata Davenp. Cat. Davenp. Herb. Suppl. 44. 1883.

Nebraska and Missouri to Arizona and New Mexico.

Notholaena fendleri Kunze, Farrnkr. 2: 87. pl. 136. 1851.

Wyoming to New Mexico and Arizona.

Notholaena tenera Gillies; Hook. Curtis's Bot. Mag. 58 : pl. 3055. 1831.

Southern Utah, Arizona, and southern California.

CHEILOGRAMMA Blume, Fl. Javae 2: 70. 1828.

Cheilogramma lanceolata (L.) Blume, Fl. Javae 2 : 70.1828.

Pteris lanceolata L. Sp. Pl. 1073. 1753.

Taenitis lanceolata R. Br. Prodr. Fl. Nov. Holl. 1 : 154. 1810.

Old Rhodes Key, Florida (Curtiss).

vitTaria J. E. Smith, Mém. Acad. Roy. Sci. Turin 5 : 413. 1793.

Vittaria lineata (L.) J. E. Smith, Mém. Acad. Roy. Sci. Turin 5 : 413. 1793. GRASS FERN.

Pteris lineata L. Sp. Pl. 1073. 1753.

Florida, as far north as Jacksonville.

ADIANTUM L. Sp. Pl. 1094. 1753.

Adiantum capillus-veneris L. Sp. Pl. 1096. 1753. Venus-HAIR Fern. BLACK MAIDENHAIR.

Virginia to Florida, west to Missouri, Utah, California, and Texas. Also in the

Black Hills, Dakota (Bessey), and New York (Rous).

Adiantum modestum Underw. Bull. Torr. Club 28 : 46. 1901.

Roswell, New Mexico (F.S. Earle). 
Adiantum tenerum Sw. Fl. Ind. Oce. $3: 1719.1806$.

Florida.

Adiantum jordani C. Müll.; Kuhn, Jahrb. Kön. Bot. Gart. Berlin 1:346. 1881.

Adiantum emarginatum Hook.; D. C. Eaton, Ferns N. Am. 1 : 285. pl. 38. f. 1-3. 1879 , not Bory.

California, New Mexico, Nevada, and Oregon.

Adiantum tricholepis Fée, $8^{\text {me }}$ Mém. Fam. Foug. 72. 1854-57.

Western Texas and New Mexico.

Adiantum pedatum L. Sp. Pl. 1095. 1753. MaIdenhaIr. *

Nova Scotia to British Columbia, south to Georgia, Mississippi, Arkansas, Kansas, Utah, and California. Also in Alaska.

PTERIS L. Sp. Pl. 1073. 1753.

Pteris longifolia L. Sp. Pl. 1074. 1753.

Florida.

Fteris cretica L. Mant. $1: 130.1767$.

Florida. Naturalized locally in Illinois.

Pteris serrulata L. f. Suppl. Pl. 445. 1781. Ribbon Fern. Spider fern. SAW-LEAVED BRACKEN.

Alabama, Georgia, and South Carolina. Probably escaped from cultivation.

PTERIDIUM Scop. Fl. Carn. ed. 1. 169. 1760.

Pteridium aquilinum (L.) Kuhn in Decken's Reisen III. Bot. OstAfrika 11. 1879. Brake. Bracken. Eagle fern. Umbrella FERN. HOG BRAKES.

Pteris aquilina L. Sp. Pl. 1075. 1753.

Newfoundland and northern Quebec to northern Alabama, Missouri, and Manitoba.

Pteridium aquilinum pseudocaudatum Clute, Fern. Bull. 8: 39. 1900, as syn.

Long Island, New York, to northern Florida, Alabama, and Texas, but mainly confined to territory near the coast.

Pteridium aquilinum pubescens Underw. Our Native Ferns ed. 6.91. 1900 .

Pteris aquilina lanuginosa of American authors. Not Pteris lanuginosa Bory; Willd. Sp. Pl. 5 : 403.1810.

Arizona and California to British Columbia.

Pteridium caudatum (L.) Maxon, comb. nov.

Pteris caudata L. Sp. Pl. 1075. 1753.

Pteris aquilina var. caudata Hook. Sp. Fil. 2 : 196. 1858.

Southern Florida.

*Includes the var. rangiferinum Burgess, Proc. Roy. Soc. Canada $4^{4}: 11.1887$, an extreme form of the Pacific coast material, the most of which probably represents a species distinct from $A$. pedatum. 
CHeilanthes Sw. Syn. Fil. 126. 1806.

Cheilanthes californica (Nutt.) Mett. Abh. Senck. Nat. Gesell. 3: 88. 1859-61. LACE FERN.

Aspidotis californica Nutt.; Hook. Sp. Fil. 2 : 71. 1858, as syn.

Hypolepis californica Hook. Sp. Fil. 2: 71. 1858.

California.

Cheilanthes amoena A. A. Eaton, Fern Bull. 5: 44. 1897. Fresno County, California.

Cheilanthes wrightii Hook. Sp. Fil. 2: 87. pl. 110. A. 1858.

Western Texas, Arizona, and New Mexico.

Cheilanthes pringlei Davenp. Bull. Torr. Club $10: 61 . p l .34 .1883$.

Southeastern Arizona.

Cheilanthes microphylla Sw. Syn. Fil. 127. 1806.

Florida, Texas, and New Mexico.

Cheilanthes alabamensis (Buckl.) Kunze, Linnaea 20:4. 1847.

Pteris alabamensis Buckl. Am. Journ. Sci. 45 : 177. 1843.

Virginia to Alabama, west to Illinois, Tennessee, Arkansas, Texas, and Arizona.

Cheilanthes viscida Davenp. Bull. Torr. Club $6: 191.1877$.

Rather widely distributed in California, though rare.

Cheilanthes leucopoda Link, Fil. Sp. Hort. Berol. 66. 1841.

Texas.

Cheilanthes lanosa (Michx.) Watt,Journ.Bot.Brit.\& Foreign 12: 48. 1874. Nephrodium lanosum Michx. Fl. Bor. Am. 2 :270. 1803.

Cheilanthes vestita Sw. Syn. Fil. 128. 1806.

Connecticut and New York to Georgia, west to Kansas, Indian Territory, and Texas.

Cheilanthes cooperae D. C. Eaton, Bull. Torr. Club 6:33. 1875.

Central and southern California. Rare.

Cheilanthes gracillima D. C. Eaton in Rep. U. S. \& Mex. Bound. Surv. $2^{1}: 234.1859$.

Cheilanthes vestita Brack. in Wilkes's U. S. Explor. Exped. 16 : 91. 1854, not Sw. British Columbia to Idaho and California.

Cheilanthes lendigera (Cav.) Sw. Syn. Fil. 128. 1806.

Pteris lendigera Cav. Descr. Pl. 268. 1802.

Huachuca Mountains, Arizona (Lemmon).

Cheilanthes feei Moore, Index Fil. xxxviii. 1857.

Myriopteris gracilis Fée, Gen. Fil. 150. 1850-52.

Cheilanthes gracilis Mett. Abh. Senck. Nat. Gesell. 3:80. 1859-61, not Kaulf. Cheilanthes lanuginosa Nutt.; Hook. Sp. Fil. 2:99. 1858, as syn.

Illinois and Minnesota to British Columbia, south to Texas, New Mexico, and Arizona.

Cheilanthes tomentosa Link, Hort. Berol. 2:42. 1833. WoolLY LIPFERN.

Virginia to Georgia, west to Missouri, Texas, and Arizona. 
Cheilanthes eatoni Baker in Hook. \& Baker, Syn. Fil. ed. 1. 140. 1868.

Cheilanthes tomentosa var. eatoni Davenp. Cat. Davenp. Herb. Suppl. 49. 1883. Arizona and Texas.

Cheilanthes fibrillosa Davenp. Bull. Torr. Club 12: 21. 1885, as syn. San Jacinto Mountains, California (Parish).

Cheilanthes parishii Davenp. Bull. Torr. Club 8:61. 1881. San Diego County, California (Parish).

Cheilanthes fendleri Hook. Sp. Fil. 2: 103. pl. 10\%. B. 1858. Texas and Colorado to California.

Cheilanthes clevelandii D. C. Eaton, Bull. Torr. Club 6:33. 1875. California.

Cheilanthes myriophylla Desv. Mag. Gesell. Nat. Fr. Berlin 5 : 328. 1811. Cheilanthes elegans Desv. Mag. Gesell. Nat. Fr. Berlin 5 : 328. 1811.

Cheilanthes villosa Davenp. Cat. Davenp. Herb. Suppl. 45. 1883.

Texas to Arizona.

Cheilanthes lindheimeri Hook. Sp. Fil. 2 : 101.pl. 10\%. A. 1858. Western Texas to Arizona.

Cheilanthes argentea (S. G. Gmel.) Kunze, Linnaea 23:242. 1850.

Pteris argentea S. G. Gmel. Nov. Comm. Acad. Sci. Petrop. 12:519. pl. 12. f. 2. 1768.

Alaska.

CRYPTOGRAmma R. Br. App. Frankl. Journ. 767. 1823.

Cryptogramma acrostichoides R. Br. App. Frankl. Journ. 767. 1823. PARSLEY FERN.

Alaska and Mackenzie south to California, Colorado, and the northern shores of Lake Huron.

Cryptogramma stelleri (S. G. Gmel.) Prantl, Engler's Bot. Jahrb. 3 : 413. 1882. Slender ClifF-BRAKE.

Pteris stelleri S. G. Gmel. Nov. Comm. Acad. Sci. Petrop. 12:519. pl. 12. f. 1. 1768.

Pellaea stelleri Watt, Can. Fil. No. 2. 1869-70.

Pteris gracilis Michx. Fl. Bor. Am. 2 : 262. 1803.

Pellaea gracilis Hook. Sp. Fil. 2: 138. pl. 133. B. 1858.

Labrador to Alaska, south to Massachusetts, Pennsylvania, Illinois, Iowa, and in the Rocky Mountains to Colorado.

PELl.AEA Link, Fil. Sp. Hort. Berol. 59. 1841.

Pellaea breweri D. C. Eaton, Proc. Am. Acad. 6 : 555. 1865.

Montana to Colorado, Nevada, Oregon, and California.

Pellaea occidentalis (E. Nelson) Rydberg, Mem. N. Y. Bot. Gard. 1:466. 1900.

Pellaea atropurpurea occidentalis E. Nelson, Fern Bull. $7: 30.1899$.

Pellaea pumila Rydberg, Mem. N. Y. Bot. Gard. 1:4. 1900.

South Dakota to Wyoming and Washington.

Pellaea atropurpurea (L.) Link, Fil. Sp. Hort. Berol. 59. 1841. PuRPLE-STEMMEd Cliff-BRAKe. Blue Fern. Winter BRAKE.

Pteris atropurpurea L. Sp. Pl. 1076. 1753. 
Massachusetts, Vermont, and Ontario to British Columbia and Mackenzie, south to Georgia, Mississippi, Texas, Arizona, and California.

Pellaea aspera (Hook.) Baker in Hook. \& Baker, Syn. Fil. ed. 1. 148. 1868. Cheilanthes aspera Hook. Sp. Fil. 2 : 111. pl. 108. A. 1858.

Western Texas and New Mexico.

Pellaea andromedaefolia (Kaulf.) Fée, Gen. Fil. 129. 1850-52. Coffee FERN.

Pteris andromedaefolia Kaulf. Enum. Fil. 188. 1824.

California and Arizona.

Pellaea pulchella (Mart. \& Gal.) Fée, Gen. Fil. 129. 1850-52. Allosorus pulchellus Mart. \& Gal. Mém. Acad. Brux. 15 $: 47.1842$.

Western Texas and New Mexico.

Pellaea marginata (H. B. K.) Baker in Hook. \& Baker, Syn. Fil. ed. 1.

151. 1868.

Cheilanthes marginata H. B. K. Nov. Gen. et Sp. Pl. $1: 22.1815$. Huachuca Mountains, Arizona (Lemmon).

Pellaea ternifolia (Cav.) Link, Fil. Sp. Hort. Berol. 59. 1841

Pteris ternifolia Cav. Descr. Pl. 266. 1802. Western Texas.

Pellaea brachyptera (Moore) Baker in Hook. \& Baker, Syn. Fil. ed. 2. 477. 1873.

Platyloma brachypterum Moore, Gard. Chron. 1873 : 141. 1873.

California and Oregon.

Pellaea ornithopus Hook. Sp. Fil. 2 : 143. pl. 116. A. 1858. BIRD's-Foot CLIFF-BRAKE. BLACK FERN.

California.

Pellaea wrightiana Hook. Sp. Fil. 2:142. pl. 115. B. 1858.

Kansas to Texas, Arizona, and California. Extremely variable.

Pellaea densa (Brack.) Hook. Sp. Fil. 2 : 150. pl. 125. B. 1858.

Onychium densum Brack. in Wilkes's U. S. Explor. Exped. 16: 120. pl. 13. f. 2. 1854.

British Columbia and Washington to Montana, Wyoming, Utah, and California. Also on Mt. Albert, Quebec, and in Grey County, Ontario (Ami).

Pellaea bridgesii Hook. Sp. Fil. 2 : 238. 1858.

California.

Pellaea flexuosa (Kaulf.) Link, Fil. Sp. Hort. Berol. 60. 1841.

Pteris flexuosa Kaulf.; Schlecht. \& Cham. Linnaea 5 : 614. 1830, excl. syn. Texas to California.

Pellaea intermedia Mett. in Kuhn, Linnaea $36: 84$. 1869-70.

Texas to Arizona.

STRUTHIOPTERIS Scop. Fl. Carn. ed. 1. 168. 1760.

Struthiopteris spicant (L.) Weiss, Pl. Crypt. 287. 1770. DEer FerN.

HARD FERN.

Osmunda spicant L. Sp. Pl. 1066. 1753.

Lomaria spicant Desv. Mag. Gesell. Nat. Fr. Berlin 5 : 325. 1811.

Blechnum boreale Sw. Schrad. Journ. Bot. 1800² : 75. 1801.

Blechnum spicant J. E. Smith, Mém. Acad. Roy. Sci. Turin 5 : 411. 1793.

California to British Columbia and Alaska. 
BLECHNUM L. Sp. Pl. 1077. 1753.

Blechnum serrulatum Richard, Act. Soc. Hist. Nat. Paris 1: 114. 1792. Florida.

W00Dwardia J. E. Smith, Mém. Acad. Roy. Sci. Turin 5 : 411. 1793.

Woodwardia virginica (L.) J. E. Smith, Mém. Acad. Roy. Sci. Turin $5: 412.1793$. ChaIN-Fern. Bog FERN.

Blechnum virginicum L. Mant. 2 : 307. 1771.

Nova Scotia to Ontario and Michigan, south to Florida, Louisiana, and Arkansas.

Woodwardia areolata (L.) Moore, Index Fil. xlv. 1857. NARRow-Leaved CHAIN-FERN

Acrostichum areolatum L. Sp. Pl. 1069. 1753.

Woodwardia angustifolia J. E. Smith, Mém. Acad. Roy. Sci. Turin 5 : 411.1793. Maine to Florida, Louisiana, and Arkansas. Also in Michigan.

Woodwardia spinulosa Mart. \& Gal. Mém. Acad. Brux. 155 : 64. 1842.

Woodwardia chamissoi Brack. in Wilkes's U. S. Explor. Exped. 16 : 138. 1854.

Woodwardia radicans var. americana Hook. Sp. Fil. 3 : 67. 1860.

California and Arizona. Also in Washington (Flett).

ASPLENIUM L. Sp. Pl. 1078. 1753.

Asplenium serratum L. Sp. Pl. 1079. 1753.

Florida.

Asplenium pinnatifidum Nutt. Gen. 2 : 251. 1818. Pinnatifid spleenWORT.

New Jersey and Pennsylvania to Georgia, Alabama, Missouri, and Arkansas. Rare and local.

Asplenium ebenoides R. R. Scott; Berkeley, Journ. Roy. Hort. Soc. 1866 : 87.1866.

Vermont to Virginia. Also in Illinois and Alabama. Extremely rare and local.

Asplenium platyneuron (L.) Oakes; D. C. Eaton, Ferns N. Am. 1: 24.

1878. Ebony spleenwort. SCREW FERN.

Acrostichum platyneuros L. Sp. Pl. 1069. 1753.

Asplenium ebeneum Ait. Hort. Kew. 3 : 462.1789.

Florida to Maine and southeastern Ontario, west to Texas and Colorado.

Asplenium parvulum Mart. \& Gal. Mém. Acad. Brux. 155 : 60. pl. 15. $f$. 3. 1842. Silall SPleEnwort. Little Ebony spleenwort.

Virginia to Florida, west to Kansas, Texas, and Arizona.

Asplenium trichomanes L. Sp. Pl. 1080. 1753. DwarF spleEnwort. Maidenhair spleenwort. Wall spleenwort. Baby Fern.

Nova Scotia and the eastern coast of Hudson Bay to Alabama, Texas, and Arizona, northwestward to Oregon, British Columbia, and Alaska.

Asplenium vespertinum Maxon, Bull. Torr. Club 27: 197. 1900.

Southern California.

Asplenium monanthes L. Mant. 1: 130. 1767.

Asplenium monanthemum L.; Murray, Syst. Veg. ed. 1. 933. 1784.

Huachuca Mountains, Arizona (Lemmon). 
Asplenium viride Huds. Fl. Angl. 385. 1762. Green splennwort.

New Brunswick, northern Vermont and Quebec to Alaska, south to Oregon,

Wyoming, and Colorado. Also in Greenland. Local.

Asplenium trichomanes-dentatum L. Sp. Pl. 1080. 1753.

Asplenium dentatum of authors.

South Carolina to Florida.

Asplenium angustifolium Michx. Fl. Bor. Am. 2 : 265. 1803. NArrow-

Leaved spleenwort. SWamp SPleenwort. Kidney fern.

Northern New England and southern Quebec to Wisconsin, south to northern Georgia, Tennessee, and Missouri.

Asplenium firmum Kunze, Bot. Zeit. 3: 283. 1845.

Florida and trizona.

Asplenium septentrionale (L.) Hoffm. Deutsch. Fl. 2 : 12. 1795. Forked SPLEENWORT.

Acrostichum septentrionale L. Sp. Pl. 1068. 1753.

Colorado, New Mexico, and Arizona; Black Hills of South Dakota (Rydberg).

Asplenium ruta-muraria L. Sp. Pl. 1081.1753. Wall Rue. Rue SPLeenWORT.

Vermont, southern Ontario, and Michigan, south to Alabama and Missouri.

Asplenium montanum Willd. Sp. Pl. $5: 342$. 1810. Mountain spleenWORT.

Connecticut, New York, and Ohio, south to Georgia, Alabama, and Arkansas.

Asplenium glenniei Baker in Hook. \& Baker, Syn. Fil. ed. 2. 488. 1873. Huachuca Mountains, Arizona (Lemmon).

Asplenium fontanum (L.) Bernh. Schrad. Journ. Bot. 1799¹ : 314. 1799. Polypodium fontanum L. Sp. Pl. 1089. 1753.

Lycoming County, Pennsylvania (McMinn); near Springfield, Ohio (Spence).

Asplenium bradleyi D. C. Eaton, Bull. Torr. Club 4:11. 1873.

New York to Georgia, Arkansas, and Missouri.

Asplenium myriophyllum (Sw.) Presl, Rel. Haenk. 1: 48. 1830.

Caenopteris myriophyllum Sw. Fl. Ind. Occ. $3: 1626.1806$.

Florida.

Asplenium cicutarium Sw. Prodr. Veg. Ind. Occ. 130. 1788.

Florida.

ATHYRIUM Roth, Tent. Fl. Germ. $3: 58.1800$.

Athyrium thelypteroides (Michx.) Desv. Mém. Soc. Linn. Paris 6: 266. 1827. Sillery SPLEENWORT.

Asplenium thelypteroides Michx. Fl. Bor. Am. 2 : 265. 1803.

Asplenium acrostichoides Sw. Schrad. Journ. Bot. $1800^{2}: 54$. 1801. Not Athyrium acrostichoideum Bory; Merat, Fl. Paris ed. 4. 1:372. 1836.

Nova Scotia and New Brunswick to Minnesota, Illinois, Alabama, and Georgia. Athyrium filix-foemina (L.) Roth. Tent. Fl. Germ. $3: 65$. 1800. LADY FERn. Female Fern.

Polypodium filix-foemina L. Sp. Pl. 1090. 1753.

Asplenium filix-foemina Bernh. Schrad. Neues Journ. Bot. $\mathbf{1}^{2}$ : 26. 1806.

Newfoundland to Keewatin and British Columbia, south to Florida, Alabama, Indian Territory, Arizona, and California. Includes a large number of interesting forms, of which several are probably of subspecific rank. 
Athyrium cyclosorum Rupr. Beitr. Pflanzenk. Russ. Reich. $3: 41.1845$. Athyrium filix-foemina var. cyclosorum Ledeb. Fl. Ross. 4:519. 1853.

Asplenium filix-foemina var. cyclosorum Rupr.; D. C. Eaton in U. S. Geog. Surv. W. 100th Merid. 6:331. 1879.

Alaska to California, Arizona, and Nebraska.

PHYLLITIS Ludwig; Inst. Hist. Phys. Reg. Veg. ed. 2. 142. 1757.

Phyllitis scolopendrium (L.) Newm. Hist. Brit. Ferns ed. 2. 10. 1844. Hart's-tongue. Caterpillar fern. Hound's-tongue fern.

Asplenium scolopendrium L. Sp. Pl. 1079. 1753.

Scolopendrium vulgare J. E. Smith, Mém. Acad. Roy. Sci. Turin 5 : 421.1793.

Scolopendrium scolopendrium Karst. Deutsch. Fl. ed. 1. 278. 1880-83.

Central New York; Tennessee; New Brunswick; Grey and Simcoe counties, Ontario. Also in Alaska (?).*

CAMPTOS0RUS Link, Hort. Berol. 2 : 69. 1833.

Camptosorus rhizophyllus (L.) Link, Hort. Berol. 2:69. 1833. WALKING LEAF. WALKING FERN. WALL LINK.

Asplenium rhizophylla L. Sp. Pl. 1078. 1753.

Maine and southern Quebec to Minnesota, south to Georgia, Alabama, and Kansas.

Camptosorus rhizophyllus intermedius Arthur, Bot. Gaz. 8: 200. pl. 3. 1883.

Iowa.

PHEG0PTERIS Fée, Gen. Fil. 242. 1850-52.

Phegopteris phegopteris (L.) Underw.; Small, Bull. Torr. Club 20 : 462. 1893. LONG BEECH FERN.

Polypodium phegopteris L. Sp. Pl. 1089. 1753.

Phegopteris polypodioides Fée, Gen. Fil. 243. 1850-52.

Newfoundland to Alaska, south to Virginia, Michigan, Iowa (Fitzpatrick), and Washington. Also in Greenland.

Phegopteris hexagonoptera (Michx.) Fée, Gen. Fil. 243. 1850-52. BROAD BEECH FERN.

Polypodium hexagonopterum Michx. Fl. Bor. Am. 2 : 271. 1803.

New England and Quebec to Minnesota, south to Kansas, Louisiana, and Florida.

Phegopteris alpestris (Hoppe) Mett. Fil. Hort. Bot. Lips. 83. 1856. ALPINE POLYPODY.

Polypodium alpestre Hoppe, Taschenb. 216. 1805.

California to Montana and British Columbia.

Phegopteris dryopteris (L.) Fée, Gen. Fil. 243. 1850-52. OAK FERN.

Polypodium dryopteris L. Sp. Pl. 1093. 1753.

Newfoundland to Alaska, south to Virginia, Minnesota, Kansas, Colorado, and

Oregon. Also in Greenland.

Phegopteris robertiana (Hoffm.) Underw. Our Native Ferns ed. 6. 109. 1900. LIMESTONE POLY PODY.

Polypodium robertianum Hoffm. Deutsch. Fl. 2: [Add. 4.]. 1795. 
Phegopteris dryopteris var. robertianum Davenp. Cat. Davenp. Herb. Suppl. 47. 1883.

Phegopteris calcarea Fée, Gen. Fil. 243. 1850-52.

Labrador to Quebec, Iowa, Minnesota, and Manitoba. Reported from Idaho.

Phegopteris tetragona (Sw.) Fée, Gen. Fil. 243. 1850-52.

Polypodium tetragonum Sw. Fl. Ind. Occ. 3:1670. 1806.

Marion County, Florida (Reynolds).

Phegopteris reptans (Sw.) D. C. Eaton, Bull. Torr. Club $10: 101.1883$.

Polypodium reptans Sw. Fl. Ind. Oce. $3: 1655.1806$.

Near Brooksville, Florida ( $J$. Donnell Smith).

DRYOPTERIS Adans. Fam. Pl. 2:20. 1763.

Dryopteris oreopteris (Sw.) Maxon, comb. nov. Heath Fern.

Aspidium oreopteris Sw. Schrad. Journ. Bot. $1800^{2}$ : 35. 1801.

Polypodium montanum J. A. Vogler, Dissert. Polyp. Mont. 1781, not Lam. 1778.

Dryopteris montana Kuntze, Rev. Gen. Pl. 2 : 813. 1891.

British Columbia (Macoun); Unalaska Island, Alaska (Turner); Washington (Elmer).

Dryopteris nevadensis (D. C. Eaton) Underw. Our Native Ferns ed. 4. 113. 1893.

Aspidium nevadense D. C. Eaton, Ferns N. Am. 1 : 73. pl. 10. 1878.

California and Oregon.

Dryopteris contermina strigosa (Fée) Underw. Our Native Ferns ed. 4. 113. 1893.

Aspidium strigosum Fée, Hist. Foug. et Lycop. Antilles 78. pl. 22. f. 2. 1866.

Aspidium conterminum var. strigosum D. C. Eaton, Bull. Torr. Club 7:62. 1880.

Florida.

Dryopteris noveboracensis (L.) A. Gray, Manual ed. 1. 630. 1848. NEw YORK FERN.

Polypodium noveboracense L. Sp. Pl. 1091. 1753.

Aspidium noveboracense Sw. Schrad. Journ. Bot. $1800^{2}: 38.1801$.

Newfoundland to Ontario and Minnesota, south to northern Georgia, Alabama, and Arkansas.

Dryopteris simulata Davenp. Bot. Gaz. $19: 497$. 1894, as syn.

Aspidium simulatum Davenp. Bot. Gaz. 19 : 495. 1894.

Maine to Maryland. Reported also from Indian Territory and Missouri. Probably of wider range.

Dryopteris thelypteris (L.) A. Gray, Manual ed 1. 630. 1848. Marsh FERN. SNUFF-BOX FERN.

Acrostichum thelypteris L. Sp. Pl. 1071. 1753.

Aspidium thelypteris Sw. Schrad. Journ. Bot. $1800^{2}: 40.1801$.

New Brunswick to Manitoba, south to Kansas, Texas, and Florida.

Dryopteris patens (Sw.) Kuntze, Rev. Gen. Pl. 2: 813.1891. Sweet FERN.

Polypodium patens Sw. Prodr. Veg. Ind. Occ. 133.1788.

Aspidium patens Sw. Syn. Fil. 49. 1806.

Aspidium molle Sw. Schrad. Journ. Bot. $1800^{2}: 34.1801$.

Florida and Alabama to California. 
Dryopteris unita (L.) Kuntze, Rev. Gen. Pl. 2 : 811. 1891.

Polypodium unitum L. Sp. Pl. ed. 2. 1546. 1764.

Aspidium unitum var. glabra Mett. Ann. Mus. Bot. Ludg. Bat. 1 : 230. 1863-64.

Not Aspidium glabrum Mett. Abh. Senck. Nat. Gesell. $2: 343$. 1856-58. Florida.

Dryopteris fragrans (L.) Schott, Gen. Fil. 1834. Fragrant Fern.

Polypodium fragrans L. Sp. Pl. 1089. 1753.

Aspidium fragrans Sw. Schrad. Journ. Bot. $1800^{2}$ : 35. 1801.

Labrador to Alaska, south to Maine, New Hampshire, Vermont, New York, Wisconsin, and Minnesota. Also in Greenland.

Dryopteris aquilonaris Maxon, Bull. Torr. Club 27: 638. 1900. Cape Nome, Alaska (Flett).

Dryopteris floridana (Hook.) Kuntze, Rev. Gen. Pl. 2 : 812. 1891.

Nephrodium floridanum Hook. Fil. Exot. pl. 99. 1859.

Aspidium floridanum D. C. Eaton in Chapm. Fl. So. U. S. ed. 1. 595. 1860.

Florida and Alabama.

Dryopteris cristata (L.) A. Gray, Manual ed. 1. 631. 1848. Crest fern. Crested shield Fern.

Polypodium cristatum L. Sp. Pl. 1090. 1753.

Aspidium cristatum Sw. Schrad. Journ. Bot. $1800^{2}$ : 37. 1801.

Newfoundland to Saskatchewan, south to Virginia, Arkansas, Nebraska, and Idaho.

Dryopteris cristata clintoniana (D. C. Eaton) Underw. Our Native Ferns ed. 4.115 .1893$.

Aspidium cristatum var. clintonianum D. C. Eaton in A. Gray, Manual ed. 5. 665. 1867.

Maine and Ontario to Wisconsin, south to Virginia (Wm. Palmer).

Dryopteris goldiana (Hook.) A. Gray, Manual ed. 1. 631.1848. GoLdie's FERN.

Aspidium goldianum Hook. Edinb. Phil. Journ. 6 : 333. 1822.

New Brunswick to Minnesota, south to North Carolina, Tennessee, and Iowa.

Dryopteris goldiana celsa Palmer, Proc. Biol. Soc. Wash. 13 : 65. pl. 1. f. 1-6. 8-12. 1899. Log FERN.

Dismal Swamp, Virginia ( Wm. Palmer; Bartsch.).

Dryopteris filix-mas (L.) Schott, Gen. Fil. 1834. Male fern. Sweet FERN.

Polypodium filix-mas L. Sp. Pl. 1090. 1753.

Aspidium filix-mas Sw. Schrad. Journ. Bot. $1800^{2}$ : 38. 1801.

Nova Scotia and Newfoundland to Alaska, south to Michigan, South Dakota, Arizona, and California.

Dryopteris cristata $\times$ marginalis Davenp. Bot. Gaz. 19: 497. 1894, as syn. Aspidium cristatum $\times$ marginale Davenp. Bot. Gaz. 19 : 494. 1894.

All the New England States and New Jersey. Probably of wider distribution.

Dryopteris marginalis (L.) A. Gray, Manual ed. 1. 632. 1848. MarGINAL SHIELD FERN. ROCK FERN. WOOD FERN.

Polypodium marginale L. Sp. Pl. 1091. 1753.

Aspidium marginale Sw. Syn. Fil. 50. 1806.

Nova Scotia to British Columbia, south to Indian Territory, Arkansas, Alabama, and Georgia. 
Dryopteris rigida arguta (Kaulf.) Underw. Our Native Ferns ed. 4. 116. 1893.

Aspidium argutum Kaulf. Enum. Fil. 242. 1824.

Aspidium rigidum var. argutum D. C. Eaton in U. S. Geog. Surv. W. 100th Merid. $6: 333.1879$.

California to Alaska, chiefly near the coast.

Dryopteris spinulosa (Retz.) Kuntze, Rev. Gen. Pl. 2 : 813. 1891. SPINy SHIELD FERN.

Polypodium spinulosum Retz. Fl. Scand. ed 2. 250. 1795.

Aspidium spinulosum Sw. Schrad. Journ. Bot. 1800² : 38. 1801.

Newfoundland to Alaska, south to Virginia, Kentucky, Nebraska, and Washington.

Dryopteris spinulosa intermedia (Muhl.) Underw. Our Native Ferns ed. 4. 116. 1893. Common SPINY Fern.

Aspidium intermedium Muhl.; Willd. Sp. Pl. 5 : 262. 1810.

Aspidium spinulosum var. intermedium D. C. Eaton in A. Gray, Manual ed. 5. 665. 1867.

Dryopteris intermedia A. Gray, Manual ed. 1.630. 1848.

Labrador to Alaska, south to North Carolina and Tennessee.

Dryopteris spinulosa dilatata (Hoffm.) Underw. Our Native Ferns ed.

4. 116. 1893. Spreading WOOD-FERn.

Polypodium dilatatum Hoffm. Deutsch. Fl. 2 : 7.1795.

Aspidium spinulosum var. dilatatum Hook. Brit. Fl. 444. 1830.

Dryopteris dilatata A. Gray, Manual ed. 1. 631. 1848.

Newfoundland to Alaska, south to California, Montana, and Virginia; mostly confined to the mountains.

Dryopteris boottii (Tuckerm.) Underw. Our Ńative Ferns ed. 4. 117. 1893. BOOTT's WOOD-FERN.

Aspidium boottii Tuckerm. Hovey's Mag. Hort. 9 : 145.1843.

Aspidium spinulosum var. boottii D. C. Eaton in A. Gray, Manual ed. 5. 665. 1867. Nova Scotia, southern Ontario, and Minnesota, south to northern Virginia.*

Dryopteris patula (Sw.) Underw. Our Native Ferns ed. 4. 117. 1893.

Aspidium patulum Sw. Kongl. Vetensk. Akad. Handl. 1817 : 74. 1817.

Huachuca Mountains, Arizona (Lemmon).

POLYSTICHUM Roth, Tent. Fl. Germ. $3: 69.1800$.

Polystichum acrostichoides (Michx.) Schott, Gen. Fil. 1834. ChristMAS FERn. Evergreen WOOd-FERn. SHIEld FERn.

Nephrodium acrostichoides Michx. Fl. Bor. Am. 2 : 267. 1803.

Aspidium acrostichoides Sw. Syn. Fil. 44. 1806.

Dryopteris acrostichoides Kuntze, Rev. Gen. Pl. 2 : 812. 1891.

Nova Scotia and New Brunswick to Wisconsin, Iowa, Mississippi, and Florida. The variable form known as var. schweinitzii (Beck), or the var. incisum A. Gray, has the range of the type.

Polystichum munitum (Kaulf.) Presl, Tent. Pterid. 83. 1836.

Aspidium munitum Kaulf. Enum. Fil. 236. 1824.

Dryopteris munita Kuntze, Rev. Gen. Pl. 2 : 813. 1891.

California, Oregon, and Idaho, northward to Alaska.

* Reported from Loring, Alaska, by Miss Grace E. Cooley in Bull. Torr. Club 19: 246. 1892. 
Polystichum munitum imbricans (D. C. Eaton) Maxon, Fern Bull. 8: 30. 1900.

Aspidium munitum var. imbricans D. C. Eaton, Ferns N. Am. 1 : 188. pl. 25. f. 3. 1878.

California to British Columbia.

Polystichum munitum inciso-serratum (D. C. Eaton) Underw. Our Native Ferns ed. 6. 116. 1900.

Aspidium munitum var. inciso-serratum D. C. Eaton, Ferns N. Am. $1: 188.1878$. California to British Columbia.

Polystichum lonchitis (L.) Roth, Tent. Fl. Germ. 3:71. 1800. Holly FERN.

Polypodium lonchitis L. Sp. Fl. 1088. 1753.

Aspidium lonchitis Sw. Schrad. Journ. Bot. $1806^{2}: 30.1801$.

Dryopteris lonchitis Kuntze, Rev. Gen. Pl. 2:813. 1891.

Arctic America to Nova Scotia, southern Ontario, Wisconsin, Montana, and Washington, and in the mountains to Utah, Colorado, and California.

Polystichum scopulinum (D. C. Eaton) Maxon, Fern Bull. 8:29. 1900. Aspidium aculeatum var. scopulinum D. C. Eaton, Ferns N. Am. 2:125. pl. 62. f. 8.1880.

Washington to eastern Idaho, south to Utah and southern California. Also in Gaspé County, Quebec.

Polystichum lemmoni Underw. Our Native Ferns ed 6. 116. 1900.

Aspidium mohrioides of American authors, not Bory, Crypt. Voy. Duperr. 267. 1828.

California to Alaska.

Polystichum californicum (D. C. Eaton) Underw. Our Native Ferns ed. 6. 116.1900 .

Aspidium californicum D. C. Eaton, Proc. Am. Acad. $6: 555.1865$.

Aspidium aculeatum var. californicum D. C. Eaton in U. S. Geog. Surv. W. 100th Merid. $6: 336.1879$.

Dryopteris aculeata var. californica (D. C. Eaton) Underw. Our Native Ferns ed. 4. 112. 1893 .

California to Washington.

Polystichum braunii (Spenner) Lawson, Fern Fl. Canada [19]. 1889. Braun's holly-Fern. Prickly-Fern.

Aspidium braunii Spenner, Fl. Frib. 1:9. 1825.

Aspidium aculeatum var. braunii Döll, Rhein. Fl. 21. 1843.

Dryopteris aculeata var. braunii (Spenner) Koch; Underw. Our Native Ferns ed. 4. 112. 1893.

Dryopteris braunii (Spenner) Underw. in Britt. \& Br. Illustr. Fl. 1:15. 1896. Nova Scotia to Alaska, south to British Columbia, Michigan, Pennsylvania, Vermont, New Hampshire, and Maine.

Polystichum aculeatum (L.) Roth, Tent. Fl. Germ. 3: 79. 1800.

Polypodium aculeatum L. Sp. Pl. 1090. 1753.

Aspidium aculeatum Sw. Schrad. Journ. Bot. 1800² : 37. 1801.

Dryopteris aculeata Kuntze, Rev. Gen. Pl. 2:812. 1891.

California to Washington.

Proc. N. M. vol. xxiii- -41 
Phanerophlebia Presl, Tent. Pterid. 84. 1836.

Phanerophlebia auriculata Underw. Bull. Torr. Club $26: 212 . p 7.359$. f. 3-4.pl. 360.f. 2. 1899.

Aspidium juglandifolium in part of recent authors, not Kunze; Klotzsch, Linnaea $20: 363.1847$.

Arizona to Texas.

TECTARIA Cav. Descr. Pl. 249. 1802.

Tectaria trifoliata (L.) Cav. Descr. Pl. 249. 1802.

Polypodium trifoliatum L. Sp. Pl. 1087. 1753.

Aspidium trifoliatum Sw. Schrad. Journ. Bot. 1800² : 30. 1801.

Dryopteris trifoliata Kuntze, Rev. Gen. Pl. 2 : 814. 1891.

Florida and western Texas.

NEPHROLEPIS Schott, Gen. Fil. 1834.

Nephrolepis exaltata (L.) Schott, Gen. Fil. 1834. Sword FErn.

Polypodium exaltatum L. Sp. Pl. ed. 2. 1548. 1763.

Aspidium exaltatum Sw. Schrad. Journ. Bot. 1800²:32. 1801.

Florida.

Nephrolepis biserrata (Sw.) Schott, Gen. Fil. 1834.

Aspidium biserratum $\mathrm{Sw}$. Schrad. Journ. Bot. $1800^{2}: 32.1801$.

Aspidium acutum Sw. Syn. Fil. 46. 1806.

Nephrolepis acuta Presl, Tent. Pterid. 79. 1836.

Southern Florida.

FILIX Adans. Fam. Pl. 2:20. 1763.

Filix bulbifera (L.) Underw. Our Native Ferns ed. 6. 119. 1900. BLaDDER-FERN.

Polypodium bulbiferum L. Sp. Pl. 1091. 1753.

Cystopteris bulbifera Bernh. Schrad. Neues Journ. Bot. $1^{2}: 26.1806$.

Newfoundland to Manitoba and Iowa, south to North Carolina, Alabama, and Arkansas. Also in Alaska.

Filix fragilis (L.) Underw. Our Native Ferns ed. 6. 119. 1900. Brittle Fern. Brittle Bladder-Fern.

Polypodium fragile L. Sp. Pl. 1091. 1753.

Cystopteris fragilis Bernh. Schrad. Neues Journ. Bot. $\mathbf{1}^{2}: 27.1806$.

Newfoundland and Labrador to Alaska, south to southern California, Arizona, Kansas, Alabama, and Georgia. Extremely polymorphic, but apparently presenting no geographic subspecies. Also in Greenland.

Filix montana (Lam.) Underw. Our Native Ferns ed. 6. 119. 1900.

Polypodium montanum Lam. Fl. Frane. 1 : 23. 1778.

Cystopteris montana Bernh. Schrad. Neues Journ. Bot. 1`: 26. 1806.

Labrador and Quebec to British Columbia and Alaska, south to the northern shore of Lake Superior. Also in Colorado. Rare. 
ONOCLEA L. Sp. Pl. 1062. 1753.

Onoclea sensibilis L. Sp. Pl. 1062. 1753. Sensitive Fern. OAKLEAVED FERN.

Newfoundland to Saskatchewan, south to Nebraska, Louisiana, and Florida. The var. obtusilobata of Torrey comprises plants of which one or more of the fertile fronds are more or less foliose.

MATteUCCIA Todaro, Syn. Pl. Acot. Vasc. Sicilia 30. 1866.

Matteuccia struthiopteris (L.) Todaro, Syn. Pl. Acot. Vasc. Sicilia 30. 1866.

Osmunda struthiopteris L. Sp. Pl. 1066. 1753.

Struthiopteris germanica Willd. Enum. Pl. Hort. Bot. Berol. 1071. 1809.

Onoclea struthiopteris Hoffm. Deutsch. Fl. 2 : 11.1795.

Nova Scotia to Virginia, west to Iowa and British Columbia.

W00Dsia R. Br. Prodr. Fl. Nov. Holl. 1: 158. 1810.

Woodsia ilvensis (L.) R. Br. Prodr. Fl. Nov. Holl. 1:158. 1810. Rusty WOODSIA.

Acrostichum ilvense L. Sp. Pl. 1071. 1753.

Labrador to Alaska, south to North Carolina, Kentucky, and Minnesota. Also in Greenland.

Woodsia alpina (Bolton) S. F. Gray, Nat. Arr. Brit. Pl. 2:17. 1821. Alpine woodsia.

Acrostichum alpinum Bolton, Fil. Brit. 76. pl. 42.1790.

Acrostichum hyperboreum Liljeb. Kongl. Vetensk. Akad. Nya Handl. 14 : 201. 1793.

Woodsia hyperborea R. Br. Prodr. Fl. Nov. Holl. 1 : 158. 1810.

Labrador to Alaska, south to Maine, Vermont, northern New York and western Ontario. Also in Greenland.

Woodsia glabella R. Br. App. Frankl. Journ. 754. 1823.

Central New York and Vermont to New Brunswick, westward in Canada to British Columbia, northward to Alaska and Mackenzie. Also in Greenland.

Woodsia scopulina D. C. Eaton, Can. Nat. II. 2 : 91.1865.

Michigan and western Ontario to British Columbia, south in the mountains to Arizona and California. Also in Alaska.

Woodsia oregana D. C. Eaton, Can. Nat. II. 2 : 90. 1865.

British Columbia and Athabasca, to Manitoba, Nebraska, Oklahoma, Colorado, Arizona, and California. Also in Wisconsin and northern Michigan.

Woodsia obtusa (Spreng.) Torr. Cat. Pl. in Geol. Rep. N. Y. 195. 1840.

Polypodium obtusum Spreng. Anleit. Kennt. Gewächse ed. 1. 3: 92.1804.

Nova Scotia to Wisconsin and Nebraska, south to Georgia, Alabama, and Texas. Also in Alaska and British Columbia. 
Woodsia obtusa plummerae (Lemmon) Maxon, comb. nov.

Woodsia plummerae Lemmon, Bot. Gaz. 7 : 6. 1882.

Woodsia obtusa var. glandulosa D. C. Eaton \& Faxon, Bull. Torr. Club 9 : 50. 1882.

New Mexico and Arizona.

Woodsia mexicana Fée, $7^{\text {me }}$ Mém. Fam. Foug. 66. 1854.

Texas, New Mexico, and Arizona.

DENNSTAEDTIA Bernh. Schrad. Journ. Bot. $1800^{2}: 124.1801$.

Dennstaedtia punctilobula (Michx.) Moore, Index Fil. xevii. 1857.

Nephrodium punctilobulum Michx. Fl. Bor. Am. 2 : 268. 1803.

Dicksonia punctiloba Hook. Sp. Fil. 1 : 79. 1846.

Dicksonia pilosiuscula Willd. Enum. Pl. Hort. Berol. 1076. 1809.

Nova Scotia and New Brunswick to Ontario and Minnesota, south to Georgia and Alabama.

Family VII. MARSILEACEAE R. Br. Prodr. Fl. Nov. Holl. 1: 166. 1810.

MARSILEA L. Sp. Pl. 1099. 1753.

Marsilea quadrifolia L. Sp. Pl. 1099. 1753.

Apparently indigenous at Bantam Lake, Litchfield County, Connecticut (Allen), though possibly adventive from Europe. Extensively introduced.

Marsilea macropoda Engelm. Am. Journ. Sci. II. 3 : 56. 1847.

Texas and New Mexico.

Marsilea uncinata A. Br. Flora 22: 300. 1839.

Louisiana; Dallas, Texas (Reverchon).

Marsilea vestita Hook. \& Grev. Icon. Fil. 2: pl. 159. 1831.

Marsiler, mucronata A. Br. Am. Journ. Sci. II. 3 : 55. text f. 2. 1847.

Arkansas and Texas to California, north to Washington, British Columbia, Montana, and South Dakota. Also in Florida.

Marsilea tenuifolia Engelm.; Kunze, Am. Journ. Sci. II. 6: 89. 1848. Pierdenales, Texas (Lindheimer); western Texas (Wright).

\section{PILULARIA L. Sp. Pl. 1100. 1753.}

Pilularia americana A. Br. Monatsb. Kön. Akad. Wiss. Berlin 1863: 435. 1863. Pillwort.

Several localities in California. Also in Arkansas (Nuttall) and Oregon (Leiberg).

Family VIII. SALVINIACEAE Reichenb. Consp. 30. 1828.

SALVINIA Adans. Fam. Pl. 2: 15. 1763.

Salvinia natans (L.) Hoffm. Deutsch. Fl. 2: 1. 1795. Salvinia.

Marsilea natans L. Sp. Pl. 1099. 1753.

Bois Brulé Bottoms, Perry County, Missouri (Demetrio); central New York (Pursh). 
AZOLLA Lam. Encyc. 1: 343. 1783.

Azolla caroliniana Willd. Sp. Pl. 5 : 541. 1810. New York to Florida, Arizona, California, and Oregon.

Azolla filiculoides Lam. Encyc. 1: 343. 1783.

California. Probably of wider range. Introduced at Springfield, Massachusetts (A. A. Eaton).

Family IX. EQUISETACEAE Michx. Fl. Bor. Am. 2: 281. 1803. EQUISETUM L. Sp. Pl. 1061. 1753.

Equisetum arvense L. Sp. Pl. 1061. 1753. Common Horse-TAIL.

Virginia to California, north to Newfoundland and Alaska. Also in Greenland.

Very variable, apparently presenting no stable subspecies.

Equisetum pratense Ehrh. Hannöv. Mag. 1784: 138. 1784.

Nova Scotia to Alaska, south to New Jersey, Minnesota, Nebraska, and Colorado.

Equisetum telmateia Ehrh. Hannöv. Mag. 1783: 287. 1783.

California to British Columbia.

Equisetum sylvaticum L. Sp. Pl. 1061. 1753. WoOd Horse-TAIL.

Newfoundland and Labrador to Alaska, south to Virginia and Nebraska. Also in Greenland.

Equisetum palustre L. Sp. Pl. 1061. 1753. Marsh Horse-tail.

Nova Scotia to Saskatchewan and Alaska, south to Maine, Connecticut, western New York, Illinois, Minnesota (?), and Washington.

Equisetum littorale Kühl.; Rupr. Beitr. Pflanzenk. Russ. Reich. 4: 91. 1845. JoINT-GRAss.

Maine and Ontario to New Jersey and Pennsylvania, west to Minnesota (Holzinger).

Equisetum fluviatile L. Sp. Pl. 1062. 1753. Swayp Horse-tall. ToadPIPES. JoINT-GRAss.

Equisetum limosum L. Sp. Pl. 1062. 1753.

Nova Scotia to Alaska, south to Virginia, Kansas, and Washington.

Equisetum ramosissimum Desf. Fl. Atl. 2: 398. 1800.

Vancouver Island, British Columbia (Lyell). Also in southern California (Davidson).

Equisetum mexicanum Milde, Verhandl. k.-k. zoolog.-bot. Gesell. 12: 1256. 1862 .

San Bernardino and Los Angeles counties, California.

Equisetum robustum A. Br. Am. Journ. Sci. 46: 88. 1844.

New Jersey to Georgia and Louisiana, west to British Columbia and California.

Equisetum hyemale L. Sp. Pl. 1062. 1753. Scouring-rush. Pipes. SHAVE-GRASS.

Quebec and New England to Virginia, Texas, California, Washington, and British Columbia.

Equisetum laevigatum A. Br. Am. Journ. Sci. 46: 87. 1844. Sноотн SCOURING-RUSH.

New Jersey to North Carolina and Louisiana, west to British Columbia, Oregon, California, and Texas. 
Equisetum variegatum Schleich. Cat. Pl. Helvet. 27. 1807.

Arctic America, south to New Brunswick, New Hampshire, New York, Nebraska, and Nevada. Also in Greenland.

Equisetum scirpoides Michx. Fl. Bor. Am. 2 : 281. 1803.

Labrador to Alaska, south to New Brunswick, Massachusetts, Pennsylvania, Illinois, Nebraska, Montana, and British Columbia. Also in Greenland.

Family X. LYCOPODLACEAE Michx. Fl. Bor. Am. 2 : 281. 1803. LYCOPODIUM L. Sp. Pl. 1100. 1753.

Lycopodium selago L. Sp. Pl. 1102. 1753. FIR CLUB-Moss.

Labrador to Alaska, south to Washington, Idaho, Michigan, northern New York, Vermont, Maine, and in the higher mountains to North Carolina. Also in Greenland.

Lycopodium porophilum Lloyd \& Underw. Bull. Torr. Club 27:150. pl. 2. f. 6-\%. 1900 .

Wisconsin; Indiana; Kentucky; Alabama (Peters; Underwood).

Lycopodium lucidulum Michx. Fl. Bor. Am. 2:284. 1803. SwayP Evergreen. Shining club-Moss.

Newfoundland and Prince Edward Island to Ontario and Minnesota, south to South Carolina, Tennessee, and Missouri.

Lycopodium inundatum L. Sp. Pl. 1102. 1753. Bog Club-Moss. Crow'sFOOT.

Newfoundland, Prince Edward Island, and Nova Scotia to New Jersey, Pennsylvania, Illinois, Michigan, and Ontario. Also from Washington to Alaska.

Lycopodium inundatum bigelovii Tuckerm. Am. Journ. Sci. 45:47. 1843.

Cape Breton Island and Nova Scotia to Vermont, Rhode Island, and New Jersey.

Lycopodium chapmani Underw., nom. nov.

Lycopodium inundatum var. appressum Chapm. Bot. Gaz. 3:20. 1878. Not Lycopodium selago var. appressum Desv. Mém. Soc. Linn. Paris $6: 180.1827$.

Lycopodium adpressum (Chapm.) Lloyd \& Underw. Bull. Torr. Club 27:153. 1900.

Lycopodium inundatum var. elongatum Chapm. Bot. Gaz. $3: 21$. 1878. Not Lycopodium elongatum Sw. Syn. Fil. 175. 1806.

Massachusetts to Rhode Island and New York (Long Island), south to Florida, Alabama, and Louisiana. Coastal in its range.

Lycopodium pinnatum (Chapm.) Lloyd \& Underw. Bull. Torr. Club $27: 155 . p l$. 3. $f .27-30 . p l .4$ in part. 1900.

Lycopodium inundatum var. pinnatum Chapm. Fl. So. U. S. ed. 1. 600. 1860.

Lycopodium alopecuroides var. pinnatum Chapm. F1. So. U. S. ed. 3. 638. 1897. Florida and Georgia to Mississippi.

\section{Lycopodium alopecuroides L. Sp. Pl. 1102. 1753.}

Long Island, New York (Clute) to Florida, Alabama, and Mississippi. Mainiy near the coast.

Lycopodium carolinianum L. Sp. PI. 1104. 1753.

New Jersey to Florida and Mississippi. 
Lycopodium annotinum L. Sp. Pl. 1103. 1753. STIFF Club-Moss.

Newfoundland and Nova Scotia to Mackenzie and Alaska, south to Massachusetts, Pennsylvania, Michigan, Minnesota, Colorado, Idaho, and Washington.

Also in Greenland. Includes the form known as var. pungens.

Lycopodium clavatum L. Sp. Pl. 1101. 1753. Running PIne. StaGHORN EVERGREEN.

Labrador and Newfoundland to Alaska, south to Oregon, Saskatchewan, Minnesota, Wisconsin, Michigan, Pennsylvania, and southern New England.

Lycopodium obscurum L. Sp. Pl. 1102. 1753. Ground PIne. Tree CLUB-MOSS.

Lycopodium dendroideum Michx. Fl. Bor. Am. 2:282. 1803.

Newfoundland and Nova Scotia to North Carolina, Tennessee, Minnesota, and Montana. Also in Alaska.

Lycopodium cernuum L. Sp. Pl. 1103. 1753.

Florida, Alabama, and Mississippi; near the coast.

Lycopodium sitchense Rupr. Beitr. Pflanzenk. Russ. Reich. 3 : 30. 1845.

Labrador to northern New England, New York, and western Ontario. Also from Idaho and Oregon to Alaska.

Lycopodium sabinaefolium Willd. Sp. Pl. 5:20. 1810. Ground Fir. Prince Edward Island, northern New England, and Ontario.

Lycopodium chamaecyparissus A. Br. in Döll, Rhein. Fl. 36.1843.

Maine to Georgia and Minnesota.

Lycopodium complanatum L. Sp. Pl. 1104. 1753.

Labrador and Cape Breton Island to British Columbia, south to Virginia, Indiana,

Minnesota and Idaho.

Lycopodium alpinum L. Sp. Pl. 1104. 1753.

British Columbia to Alaska. Also in Greenland.

PSILOTUM Sw. Syn. Fil. 187. 1806.

Psilotum nudum (L.) Griseb. Abh. Kön. Gesell. Wiss. Göttingen $7: 278$. 1857.

Lycopodium nudum L. Sp. Pl. 1100. 1753.

Bluffton, South Carolina (Mellichamp); southern Florida.

Family XI. SELAGINELLACEAE Underw. Our Native Ferns ed. 1. 103.1881.

SELAGINELla Beauv. Prodr. Aetheog. 101. 1805.*

Selaginella selaginoides (L.) Link, Fil. Sp. Hort. Berol. 158. 1841.

Lycopodium selaginoides L. Sp. Pl. 1101. 1753.

Selaginella spinosa Beauv. Prodr. Aetheog. 112. 1805.

Labrador to New Hampshire, New York, Michigan, Saskatchewan, and Alaska.

Also in Colorado and Greenland.

Selaginella rupestris (L.) Spring in Mart. Fl. Bras. 1²: 118. 1840.

Lycopodium rupestre L. Sp. Pl. 1101. 1753.

New England and Ontario to Georgia, west to California, Idaho, and British Columbia.

* A number of new species of the rupestris group have recently been described by Dr. G. Hieronymus, from western North America. (Hedwigia 39: 290 et seq. 1900). Although several of these will probably prove valid, it has been thought best, on account of insufficient material, to exclude all from the list. 
Selaginella rupestris fendleri Underw. Bull. Torr. Club 25: 127. 1898.

New Mexico and Colorado.

Selaginella densa Rydberg, Mem. N. Y. Bot. Gard. 1: 7. 1900.

Western Nebraska to Montana.

Selaginella bryoides (Nutt.) Underw. Our Native Ferns ed. 6. 138. 1900.

Lycopodium bryoides Nutt.; Baker, Handbook Fern Allies 35. 1887.

Selaginella cinerascens A. A. Eaton, Fern Bull. 7 : 33. 1899.

Southern California.

Selaginella watsoni Underw. Bull. Torr. Club 25: 127. 1898.

High mountains of Utah, Nevada, and California.

Selaginella mutica D. C. Eaton in Underw. Bull. Torr. Club 25:128. 1898. New Mexico, Arizona, and Colorado.

Selaginella tortipila A. Br. Ann. Sci. Nat. V. $3: 271.1865$.

Macon County (J. Donnell Smith) and Broad River (Rugel), North Carolina; Caesars Head, South Carolina (J. Donnell Smith).

Selaginella struthioloides (Presl) Underw. Bull. Torr. Club $25: 132$. 1898.

Lycopodium struthioloides Presl, Rel. Haenk. 1 : 82. 1830.

Selaginella oregana D. C. Eaton in Brewer \& Wats. Bot. Cal. 2 :350. 1880. Oregon and Washington.

Selaginella arenicola Underw. Bull. Torr. Club 25 : 541. 1898.

Selaginella arenaria Underw. Bull. Torr. Club $25: 129$. 1898, not Baker.

- Florida and Texas.

Selaginella rupincola Underw. Bull. Torr. Club 25 : 129. 1898.

New Mexico and Arizona.

Selaginella bigelovii Underw. Bull. Torr. Club 25 : 130. 1898.

Southern California.

Selaginella douglassii (Hook. \& Grev.) Spring, Mém. Acad. Brux. $24^{1}$ : 92. 1850 .

Lycopodium ovalifolium Hook. \& Grev. Icon. Fil. 2 : pl. 17\%. 1831, not Desv.

Lycopodium douglassii Hook. \& Grev. Bot. Misc. 2 : 396.1832.

Northern California to British Columbia.

Selaginella apus (L.) Spring in Mart. Fl. Bras. $1^{2}: 119.1840$.

Lycopodium apodum L. Sp. Pl. 1105. 1753.

Maine and Ontario to British Columbia, south to Florida, Alabama, Louisiana, and Texas.

Selaginella ludoviciana A. Br. Ann. Sci. Nat. IV. $13: 58.1860$.

Louisiana (Drummond); Alabama (Mohr); Florida (Curtiss).

Selaginella lepidophylla (Hook. \& Grev.) Spring, Mém. Acad. Brux. $24^{1}$ : 72.1850.

Lycopodium lepidophyllum Hook. \& Grev. Bot. Misc. 3 : 106. 1832. Texas to Arizona.

Selaginella pringlei Baker, Handbook Fern Allies 88. 1887.

Chenate Mountains, Texas (Nealley).

Selaginella pilifera A. Br. Ind. Sem. Hort. Berol. App. 1857: 20. 1857.
Texas (Wright). 
Family XII. ISOETACEAE Torr. Fl. State N. Y. 2: 514. 1843.

ISOETES L. Sp. Pl. 1100. 1753. QuHLwort.

Isoetes lacustris L. Sp. Pl. 1100. 1753.

Labrador to the Lake Superior region and New Jersey.

Isoetes paupercula (Engelm.) A. A. Eaton, comb. nov.

Isoetes lacustris var. paupercula Engelm. Trans. St. Louis Acad. Sci. 4:377. 1882.

Isoetes occidentalis Henderson, Bull. Torr. Club 27 : 358. 1900.

Colorado to California, Washington, and Idaho.

Isoetes heterospora A. A. Eaton, Fernwort Papers 8. 1900.

Jordan Pond, Mount Desert Island, Maine (Rand).

Isoetes riparia Engelm.; A. Br. Flora 29:178. 1846.

Along the banks of the Delaware River in the following localities: Near Philadelphia, Pennsylvania (Zantzinger; Durand)*; Camden, New Jersey (Parker; D. C. Eaton); Chester, Pennsylvania (T. C. Palmer); Wilmington, Delaware (Commons; Canby).

Isoetes tuckermani A. Br. in A. Gray, Manual ed. 5. 676. 1867.

Maine, New Hampshire, Massachusetts, and Connecticut.

Isoetes tuckermani borealis A. A. Eaton, Fernwort Papers 10. 1900.

Labrador (Allen); Epping, New Hampshire (A. A. Eaton); Mount Desert Island, Kennebago Lake (Coville), and Oldtown (Harvey), Maine.

Isoetes hieroglyphica A. A. Eaton, Fernwort Papers 10. 1900.

Maine: St. Francis Lake; Moosehead Lake; Rangeley Lakes.

Isoetes harveyi A. A. Eaton, Fernwort Papers 11. 1900.

Oldtown and Mount Desert Island, Maine; Cambridge, Massachusetts (Boott).

Isoetes foveolata A. A. Eaton in Dodge, Ferns \& Fern Allies New Eng. 38. 1896.

Epping, West Epping, East Kingston, and Newmarket, New Hampshire (A. A. Eaton); Meriden, Connecticut (F. W. Hall).

Isoetes saccharata Engelm. in A. Gray, Manual ed. 5. 676. 1867.

Banks of the Wicomico River, below Salisbury, and of the Nanticoke River, Maryland (Canby); Elk River, Maryland (T. C. Palmer) ; Four-Mile Run, near Washington, D. C. (Steele).

Isoetes saccharata reticulata A. A. Eaton in Steele, Proc. Biol. Soc. Wash. $14: 49.1901$.

Hunting Creek, near Alexandria, Virginia (Coville \& Vasey; Maxon).

Isoetes saccharata palmeri A. A. Eaton in Steele, Proc. Biol. Soc. Wash. $14: 49.1901$.

Lloyds Creek, Maryland (T. C. Palmer); Mount Vernon, Virginia (Coville).

Isoetes melanospora Engelm. Trans. St. Louis Acad. Sci. 3 : 395. 1877.

Georgia: Stone Mountain, (Canby; Underwood; Boynton); Little Stone Mountain, Lithonia (Small).

* Locality now apparently destroyed by improvements.-A. A. Eaton.

†The Robinson \& Schrenk, Quiddy Viddy Lake, Newfoundland, specimens are probably to be referred here.-A. A. Eaton. 
Isoetes echinospora braunii (Durieu) Engelm. in A. Gray, Manual ed. 5. 676. 1867.

Isoetes braunii Durieu, Bull. Soc. Bot. France 11 : 101.1864.

Isoetes echinospora var. boottii Engelm. in A. Gray, Manual ed. 5.676. 1867.

Labrador and Greenland to Alaska, southward to New England, New Jersey,

Pennsylvania, Michigan, Utah, and southern Washington.

Isoetes echinospora robusta Engelm. Trans. St. Louis Acad. Sci. 4:380. 1882 .

Isle La Motte in Lake Champlain (Pringle); Epping, New Hampshire $(A . A$. Eaton).

Isoetes echinospora muricata (Durieu) Engelm. in A. Gray, Manual ed. 5. 676. 1867.

Isoetes muricata Durieu, Bull. Soc. Bot. France $11: 100.1864$.

Near the St. John at Madawaska, Maine (Fernald); Rockingham County, New

Hampshire (A. A. Eaton); near Boston, Massachusetts; Lyme, Connecticut (C. B. Graves); Toms River, New Jersey (Parker \& Smith; Pollard).

Isoetes bolanderi Engelm. in Parry, Am. Nat. 8: 214. 1874.

Montana and Washington to western Colorado, Utah, and California.

Isoetes pygmaea Engelm. in Parry, Am. Nat. 8: 214. 1874.

Mono Pass, California (Bolander).

Isoetes macounii A. A. Eaton, Fern Bull. 8 : 12.1900.

Atka Island, Alaska (Macoun).

Isoetes maritima Underw. Bot. Gaz. 13: 94. 1888.

Alberni, Vancouver Island, British Columbia (Macoun).

Isoetes canadensis (Engelm.) A. A. Eaton, comb. nov.

Isoetes riparia var. canadensis Engelm. Trans. St. Louis Acad. Sci. 4:383. 1882.

Isoetes dodgei A. A. Eaton, Fern Bull. $6: 6.1898$.

Hastings County, Ontario (Macoun); East Wilton (Fernald), Cornish (Chickering), and Kennebunk, Maine; Uxbridge (Robbins), and Dedham (Faxon), Massachusetts; Brattleboro, Vermont (Frost); Kingston, New Hampshire (A. A. Eaton); Point Pleasant (Best), and Bethlehem, Pennsylvania.

Isoetes eatoni Dodge, Ferns \& Fern Allies New Eng. 39. 1896.

Several localities in Rockingham County, New Hampshire. Also in Essex County, Massachusetts.

Isoetes gravesii A. A. Eaton, Fernwort Papers 14. 1900.

Connecticut: Goshen (Underwood); Lyme (Graves).

Isoetes engelmanni A. Br. Flora 29:178. 1846.

Maine to Delaware and Pennsylvania. Also in Illinois and Missouri.

Isoetes engelmanni caroliniana A. A. Eaton, Fern Bull. 8:60. 1900.

Several localities in North Carolina.

Isoetes engelmanni valida Engelm. in A. Gray, Manual ed. 5. 677. 1867. Warriors Mark, Cornwall, and Smithville, Pennsylvania (Porter); Wilmington,

Delaware (Canby); Salt Pond Mountain, Virginia (Canby); Great Falls, Maryland ( Ward).

Isoetes engelmanni georgiana Engelm.Trans. St. Louis Acad. Sci. $4: 384$. 1882.

Georgia: Floyd County (Canby); Whitfield County (Harper). 
Isoetes howelli Engelm. Trans. St. Louis Acad. Sci. 4 : 385.1882.

Isoetes nuda Engelm. Trans. St. Louis Acad. Sci. 4 : 385. 1882.

Isoetes underwoodii Henderson, Bot. Gaz. 23 : 124. 1897.

Trinity and Calaveras counties, California; Dalles of the Columbia and Hood River, Oregon (Howell); Klickitat and Spokane counties, Washington (Suksdorf); Idaho (Henderson).

Isoetes flaccida Shuttlew.; A. Br. Flora 29:178. 1846.

Isoetes flaccida var. chapmani Engelm. Trans. St. Louis. Acad. Sci. 4 : 386.1882.

Several localities in Florida.

Isoetes melanopoda J. Gay, Bull. Soc. Bot. France 11: 102. 1864.

Several localities in Illinois. Clifton, Iowa (Vasey); Oklahoma (Butler); Exeter,

Nebraska; Jackson County, Missouri.

Isoetes melanopoda pallida Engelm. Trans. St. Louis Acad. Sci. 4: 387. 1882.

Texas: Dallas (Reverchon); Hempstead and Houston (Hall); Harrisburg (Joor); Columbia (Bush); Hockley ( Thurow).

Isoetes butleri Engelm. Bot. Gaz. $3: 1.1878$.

Missouri, Oklahoma, and Tennessee.

Isoetes butleri immaculata Engelm. Trans. St. Louis. Acad. Sci. 4:388. 1882.

Near Nashville, Tennessee (Gattinger); St. Louis, Missouri (Eggert).

Isoetes minima A. A. Eaton, Fern Bull. $6: 30.1898$.

Near Waverly, Spokane County, Washington (Suksdorf).

Isoetes nuttallii A. Br. in Parry, Am. Nat. 8 : 215. 1874.

Isoetes suksdorfii Baker, Handbook Fern Allies 132. 1887.

Washington, Oregon, and Idaho; Vancouver Island, British Columbia (Macoun);

Marin County, California (Mrs. Brandegee).

Isoetes orcuttii A. A. Eaton, Fern Bull. 8:13. 1900.

San Diego, California (Orcutt).

Addition to Bibliography on page 623.

1893. The Altitudinal Distribution of the Ferns of the Appalachian Mountain System. John K. Small in Bulletin of the Torrey Botanical Club $20: 455-467$. 


\section{$2 \mathrm{BHL}$ Biodiversity Heritage Library}

Maxon, William R. 1901. "A list of the ferns and fern allies of North America north of Mexico, with principal synonyms and distribution." Proceedings of the United States National Museum 23(1226), 619-651. https://doi.org/10.5479/si.00963801.1226.619.

View This Item Online: $\underline{\text { https://www.biodiversitylibrary.org/item/32360 }}$

DOI: https://doi.org/10.5479/si.00963801.1226.619

Permalink: https://www.biodiversitylibrary.org/partpdf/26647.

\section{Holding Institution}

Smithsonian Libraries

\section{Sponsored by}

Smithsonian

\section{Copyright \& Reuse}

Copyright Status: NOT_IN_COPYRIGHT

This document was created from content at the Biodiversity Heritage Library, the world's largest open access digital library for biodiversity literature and archives. Visit BHL at https://www.biodiversitylibrary.org. 